\title{
Numerical implementation of isolated horizon boundary conditions
}

\author{
José Luis Jaramillo* \\ Laboratoire de l'Univers et de ses Théories, UMR 8102 du C.N.R.S., Observatoire de Paris, F-92195 Meudon Cedex, France \\ and Instituto de Astrofísica de Andalucía, CSIC, Apartado Postal 3004, Granada 18080, Spain \\ Marcus Ansorg ${ }^{\dagger}$ \\ Max-Planck-Institut für Gravitationsphysik, Albert-Einstein-Institut, Am Mühlenberg 1, D-14476 Golm, Germany \\ François Limousin \\ Laboratoire de l'Univers et de ses Théories, UMR 8102 du C.N.R.S., Observatoire de Paris, F-92195 Meudon Cedex, France \\ and Center for Radiophysics and Space Research, Cornell University, Ithaca, New York, 14853, USA
}

(Received 2 October 2006; published 12 January 2007)

\begin{abstract}
We study the numerical implementation of a set of boundary conditions derived from the isolated horizon formalism, and which characterize a black hole whose horizon is in quasiequilibrium. More precisely, we enforce these geometrical prescriptions as inner boundary conditions on an excised sphere, in the numerical resolution of the conformal thin sandwich equations. As main results, we first establish the consistency of including in the set of boundary conditions a constant surface gravity prescription, interpretable as a lapse boundary condition, and second we assess how the prescriptions presented recently by Dain $e t$ al. for guaranteeing the well-posedness of the conformal transverse traceless equations with quasiequilibrium horizon conditions extend to the conformal thin sandwich elliptic system. As a consequence of the latter analysis, we discuss the freedom of prescribing the expansion associated with the ingoing null normal at the horizon.
\end{abstract}

DOI: 10.1103/PhysRevD.75.024019

PACS numbers: 04.25.Dm, 04.20.Ex, 04.70.Bw

\section{INTRODUCTION}

The general study of spacetimes containing a black hole whose horizon is in quasiequilibrium is of direct interest in astrophysics and numerical relativity. A particularly important application is the determination of inner boundary conditions for the construction of astrophysically realistic excised initial data for binary black holes in quasicircular orbits [1-6]. Beyond the construction of initial data, the analysis of these boundary conditions also provides helpful insight into the evolution problem. More precisely, a generalization of these conditions constitutes an integral part of the modelling of black hole horizons as world tubes of marginally trapped surfaces, according to the characterizations in the quasilocal horizon formalisms of trapping and dynamical horizons (see reviews $[7,8]$ ). In this sense, the quasiequilibrium conditions discussed here offer a test ground for the general dynamical case, in a better controlled scenario. Lessons acquired in the quasiequilibrium case can then be applied to the evolution of an excised black hole in a constrained scheme like the one proposed in Ref. [9]. In addition, quasiequilibrium conditions themselves are of direct interest in slow evolution schemes, like the minimal no-radiation approximation proposed in Ref. [10].

The isolated horizon formalism developed by Ashtekar et al. (see Ref. [7] for a review) provides a particularly

\footnotetext{
*Electronic address: jarama@iaa.es

Electronic address: marcus.ansorg@aei.mpg.de

\#Electronic address: limousin@astro.cornell.edu
}

well-suited framework for studying a black hole in equilibrium inside a generically dynamical spacetime. ${ }^{1}$ It is an example of a trapping horizon in which the horizon world tube is a null hypersurface. This null-like character encodes the quasiequilibrium characterization.

First derivations of quasiequilibrium horizon boundary conditions were presented in Refs. [2,4] (see also Ref. [3]). The detailed analysis of isolated horizons in a $3+1$ description of spacetime permits the systematization and extension of these results. Following this line of research, an ensemble of boundary conditions for the $3+1$ fields has been proposed in Refs. [11-13]. In this paper we address the problem of testing numerically these sets of boundary conditions. Translating Einstein equations into a specific system of partial differential equations, for which the isolated horizon prescriptions become actual analytical boundary conditions, requires the choice of a particular resolution scheme for the geometrical field equations. For concreteness (and also motivated by the fully constrained scheme in Ref. [9], in which constraints are solved at each time step), we focus here on the construction of initial data in a conformal thin sandwich (CTS) approach [14,15]. Making use of an excision technique, i.e. removing a sphere $\mathcal{S}$ of coordinate radius $r_{H}$ from the initial spatial slice $\Sigma$, and requiring that $\mathcal{S}$ be a spacelike slice of an

\footnotetext{
${ }^{1}$ Throughout the paper we abuse the language and use the expression black hole spacetime to refer to a spacetime containing an isolated horizon inside, without any reference to the notion of event horizon.
} 
isolated horizon, an ensemble of inner boundary conditions for the CTS elliptic system is determined. Note that throughout the paper we use dimensionless physical and geometrical quantities which result from the corresponding dimensional quantities by rescaling with the appropriate power of $r_{H}$.

Regarding index notation, Greek letters denote Lorentzian indices and are mainly used in Sec. II, where null and spacelike geometries appear in the same context. Latin indices refer specifically to objects living on spacelike slices and are used from Sec. III on.

\section{BOUNDARY CONDITIONS: GEOMETRICAL FORM}

\section{A. Geometrical boundary conditions}

In order to introduce a characterization of the term quasiequilibrium, we need a notion of time evolution. A natural evolution vector on a null world tube $\mathcal{H}$, sliced by a given family of trapped surfaces $\left\{\mathcal{S}_{t}\right\}$, is provided by the null-vector $\ell$ that Lie-drags $\mathcal{S}_{t}$ onto $\mathcal{S}_{t+\delta t}$. We consider the horizon slicing $\left\{\mathcal{S}_{t}\right\}$ as induced by a $3+1$ spacetime foliation of spacelike surfaces $\Sigma_{t}$. First, we fix the notation. We denote by $n^{\alpha}$ the timelike unit normal to $\Sigma_{t}$, by $N$ the associated lapse, and by $s^{\alpha}$ the unit spacelike normal to $\mathcal{S}_{t}$ lying on $\Sigma_{t}$. The horizon evolution vector is then expressed as $\ell^{\alpha}=N \cdot\left(n^{\alpha}+s^{\alpha}\right)$. The ingoing null-vector $k^{\alpha}$ (in the plane defined by $n^{\alpha}$ and $s^{\alpha}$ and normalized as $\left.k^{\mu} \ell_{\mu}=-1\right)$ is written as $k^{\alpha}=\frac{1}{2 N}\left(n^{\alpha}-s^{\alpha}\right)$. We denote by $\left(\gamma_{\alpha \beta}, K^{\alpha \beta}\right)$ the 3-metric on $\Sigma_{t}$ and the extrinsic curvature (with sign convention $K_{\alpha \beta}=-\frac{1}{2} \mathcal{L}_{n} \gamma_{\alpha \beta}=-\gamma_{\alpha}^{\mu} \nabla_{\mu} n_{\beta}$ ). The induced metric on the marginally trapped surface $\mathcal{S}_{t}$ is then given by $q_{\alpha \beta}=\gamma_{\alpha \beta}-s_{\alpha} s_{\beta}$. Quasiequilibrium boundary conditions follow from prescribing certain $3+1$ fields to be time independent on the horizon. In addition, other relevant boundary conditions (not necessarily related to being in quasiequilibrium) follow from: (i) analytical requirements on the well-posedness of the elliptic system, (ii) numerical control of the horizon slicing taking into account the geometry of the horizon, and (iii) choice of a coordinate system adapted to the horizon (ultimately motivated by numerical considerations). We briefly review this ensemble of conditions (for their systematic derivations and justifications, see Refs. [11-13]).

\section{B. Quasiequilibrium conditions}

Prescribing the time independence of a particular combination of $3+1$ fields can represent either an actual restriction on the geometry of $\mathcal{H}$ as a spacetime hypersurface, or it can refer to the manner in which $\mathcal{H}$ is described in the $3+1$ slicing. Both cases are relevant in a numerical relativity context.

(1) The minimal notion of quasiequilibrium is provided by the nonexpanding horizon condition, namely, the time independence of the induced metric on $\mathcal{S}$ : $q_{\alpha}^{\mu} q_{\beta}^{\nu} \mathcal{L}_{\ell} q_{\mu \nu}=0$. Expressed in terms of the expan$\operatorname{sion} \theta_{(\ell)}$ and shear $\sigma_{(\ell)}$ associated with the outgoing null normal $\ell^{\alpha}$, i.e.

$$
\begin{gathered}
\theta_{(\ell)}=q^{\mu \nu} \nabla_{\mu} \ell_{\nu}, \\
\left(\sigma_{(\ell)}\right)_{\alpha \beta}=q_{\alpha}^{\mu} q_{\beta}^{\nu} \nabla_{\mu} \ell_{\nu}-\frac{1}{2} \theta_{(\ell)} q_{\alpha \beta},
\end{gathered}
$$

this amounts to $([4,11,16]$; see also Ref. [2] for a heuristic discussion on the vanishing of the shear)

$$
\theta_{(\ell)}=0=\left(\sigma_{(\ell)}\right)_{\alpha \beta} .
$$

These three conditions $\left[\sigma_{(\ell)}\right.$ is a symmetric traceless tensor on $S^{2}$ ] constitute a true restriction of the geometry of $\mathcal{H}$, essentially related to its nullcharacter via the Raychaudhuri equation. Physically, they imply that the area of the horizon remains constant in time.

(2) Another proposed quasiequilibrium condition [11] consists in prescribing the time- independence of the vertical (i.e. in the $\ell^{\alpha}$ direction) component of the angular variation of the null normal $\ell^{\alpha}$. Explicitly this translates into $\mathcal{L}_{\ell} \Omega_{\alpha}=0$, where $\Omega_{\alpha}:=$ $-q_{\alpha}^{\mu}\left(k_{\nu} \nabla_{\mu} \ell^{\nu}\right)$. Recasting $\Omega_{\alpha}$ in terms of $3+1$ fields, this condition means that the combination $\Omega_{\alpha}={ }^{2} D_{\alpha} \ln N-K_{\mu \nu} s^{\mu} q_{\alpha}^{\nu}$ remains constant in time (where ${ }^{2} D$ is the connection associated with $\left.q_{\alpha \beta}\right)$. Its underlying justification uses the weakly isolated horizon notion (see Ref. [11]). However, we can heuristically motivate it in two manners:

(i) Given $\mathcal{S}_{t}$ with an axial symmetry generated by $\phi^{\alpha}$ and with volume element ${ }^{2} \boldsymbol{\epsilon}=\sqrt{q} d x^{a} \wedge$ $d x^{b}$, an angular momentum $J_{\mathcal{H}}=-\frac{1}{8 \pi G} \times$ $\int_{\mathcal{S}_{t}} \Omega_{\mu} \phi^{\mu 2} \epsilon$ can be associated with the horizon [17]. The surface density of this momentum, $\Omega_{\alpha}$, satisfies a Navier-Stokes-like evolution equation for a viscous fluid (see $[13,18]$ for a discussion of each term):

$$
\begin{aligned}
\mathcal{L}_{\ell} \Omega_{\alpha}+\theta \Omega_{\alpha}= & 8 \pi q^{\mu}{ }_{\alpha} T_{\mu \nu} \ell^{\nu}+{ }^{2} D_{\alpha} \kappa \\
& -{ }^{2} D_{\mu} \sigma^{\mu}{ }_{\alpha}+\frac{1}{2}{ }^{2} D_{\alpha} \theta,
\end{aligned}
$$

where $\kappa$ is the nonaffinity coefficient of the null geodesic generated by $\ell^{\alpha}: \nabla_{\ell} \ell^{\alpha}=\kappa \ell^{\alpha}$. Nonexpanding conditions in Eq. (3) imply the vanishing of the viscous terms in Eq. (4) (as well as the force surface density term; see [13]), which reduces then to the Euler equation, $\mathcal{L}_{\ell} \Omega_{\alpha}={ }^{2} D_{\alpha} \kappa$. A natural quasiequilibrium condition in this fluid analogy is given by $\mathcal{L}_{\ell} \Omega_{\alpha}=0$, which translates into: ${ }^{2} D_{\alpha} \kappa=$ 0 . This expresses the constancy of $\kappa$ on $\mathcal{S}$. Writing then $\kappa=\kappa_{\mathrm{o}}=$ const, its $3+1 \mathrm{de}-$ composition provides an evolution equation 
for the lapse:

$$
\mathcal{L}_{\ell} \ln N=\kappa_{\mathrm{o}}-s^{\mu} D_{\mu} N+N K_{\mu \nu} s^{\mu} s^{\nu}
$$

Under the gauge choice $\mathcal{L}_{\ell} N=0$, this equation becomes the boundary condition proposed in Ref. [11]:

$$
\kappa_{\mathrm{o}}=s^{\mu} D_{\mu} N-N K_{\mu \nu} s^{\mu} s^{\nu}
$$

This condition does not restrict the geometry (and therefore the physical features) of the horizon, but rather chooses a convenient $3+$ 1 description.

(ii) From the relation above, $\mathcal{L}_{\ell} \Omega_{\alpha}={ }^{2} D_{\alpha} \kappa$, one can motivate the time independence of $\Omega_{\alpha}$ from the Hamiltonian analysis of isolated horizons (see [7,19]). In this context, the nonaffinity coefficient $\kappa$ is interpreted as a surface gravity of the horizon. The constancy of $\kappa$ turns out to be a very natural equilibrium condition as the quasilocal zeroth law of black hole mechanics. In addition, such a Hamiltonian analysis provides a preferred time evolution vector on the horizon. Associated with this vector there is a canonical constant value for the surface gravity, namely $\kappa_{\mathrm{O}}=\kappa_{\text {Kerr }}(a, J)$, where $a$ and $J$ are the area and angular momentum of $\mathcal{S}_{t}$, and $\kappa_{\text {Kerr }}(a, J)$ is the corresponding surface gravity of a Kerr black hole.

\section{Other geometrical boundary conditions}

Together with quasiequilibrium motivations, some geometrical inner boundary conditions follow from genuine numerical motivations. As a first instance of such boundary conditions, in some numerical schemes it is important to keep the location of the horizon fixed at a given position. Geometrically this means that the $3+1$ evolution vector $t^{\alpha}=N n^{\alpha}+\beta^{\alpha}$ (where $\beta^{\alpha}$ is the shift vector) must be tangent to the horizon hypersurface $\mathcal{H}$. Decomposing the shift in its normal and tangential parts to $\mathcal{S}$, i.e. $\beta^{\alpha}=$ $b s^{\alpha}-V^{\alpha}$ (with $V^{\mu} s_{\mu}=0$ ), it follows $t^{\alpha}=\ell^{\alpha}-V^{\alpha}+$ $(b-N) s^{\alpha}$. Therefore $t^{\alpha}$ is tangent to $\mathcal{H}$ if and only if

$$
b-N=0 .
$$

A second example of a numerically motivated boundary condition follows from the need of a prescription for the lapse that incorporates information about the geometrical content of the horizon, but still leaves some rescaling freedom to control the size of $N$. In other words, a geometrical prescription for the slicing $\left\{\mathcal{S}_{t}\right\}$ of $\mathcal{H}$, rather than for $N$ itself. In Ref. [20] it is shown that specifying ${ }^{2} D^{\mu} \Omega_{\mu}$, i.e. the divergence of the 1 -form $\Omega_{\alpha}$ introduced above, fixes the slicing of $\mathcal{H}$ in an intrinsic manner (see also Ref. [13] for a $3+1$ discussion). From the $3+1$ expression of $\Omega_{\alpha}$ it follows that

$$
{ }^{2} \Delta \ln N={ }^{2} D^{\rho}\left(q_{\rho}^{\mu} K_{\mu \nu} s^{\nu}\right)+{ }^{2} D^{\mu} \Omega_{\mu},
$$

that fixes $\ln N$ up to a constant. As a consequence, the lapse is specified modulo a multiplicative constant that can be chosen to keep the slicing under numerical control.

\section{CONFORMAL THIN SANDWICH DECOMPOSITION}

\section{A. CTS equations}

Fixing a representative ${ }^{2} \tilde{\gamma}_{i j}$ in the conformal class of $\gamma_{i j}$, we perform the following decomposition of the 3-metric and the extrinsic curvature

$$
\gamma_{i j}=\Psi^{4} \tilde{\gamma}_{i j}, \quad K^{i j}=\Psi^{-4}\left(\tilde{A}^{i j}+\frac{1}{3} K \tilde{\gamma}^{i j}\right),
$$

where

$$
\tilde{A}^{i j}=\frac{1}{2 N}\left[(\tilde{L} \beta)^{i j}+\dot{\tilde{\gamma}}^{i j}\right], \quad K=\gamma^{i j} K_{i j},
$$

with $\dot{\tilde{\gamma}}^{i j}:=\mathcal{L}_{t} \tilde{\gamma}^{i j}$, and

$$
(\tilde{L} \beta)^{i j}=\left(\tilde{D}^{i} \beta^{j}+\tilde{D}^{j} \beta^{i}-\frac{2}{3} \tilde{D}_{k} \beta^{k} \tilde{\gamma}^{i j}\right),
$$

where $\tilde{D}_{i}$ is the Levi-Civita connection associated with $\tilde{\gamma}_{i j}$. Inserting this decomposition in the Hamiltonian and momentum constraints, and prescribing on $\Sigma$ the time derivative of the trace of the extrinsic curvature $\left(\dot{K}:=\mathcal{L}_{t} K\right)$, results in the CTS equations. This is an elliptic system for $\left(\Psi, \beta^{i}, N\right)$, once the initial free data $\left(\tilde{\gamma}_{i j}, K, \dot{\tilde{\gamma}}^{i j}, \dot{K}\right)$ are prescribed on the initial $\Sigma$. If a sphere $\mathcal{S}$ is excised in $\Sigma$, as is the case here, the boundary conditions for $\left(\Psi, \beta^{i}, N\right)$ on $\mathcal{S}$ are also a part of the free data. For concreteness, in this work we focus on free data ${ }^{3}$ with $\dot{\tilde{\gamma}}^{i j}=0=\dot{K}$, and consider different choices for $\left(\tilde{\gamma}_{i j}, K\right)$. Vacuum CTS equations, after a conformal rescaling of the lapse $N=\Psi^{a} \tilde{N}$, have the form

\footnotetext{
${ }^{2}$ We use Latin indices from now on to emphasize that equations are defined on a spacelike slice $\Sigma$.

${ }^{3}$ This choice is used in the literature $[1,2,4-6]$ as a quasiequilibrium prescription in the bulk. Gauge horizon condition (7) then has a geometrical meaning, since it links bulk and horizon quasiequilibrium notions by making the evolution vector $t^{\alpha}$ tangent to the horizon.
} 


$$
\begin{gathered}
\tilde{\Delta} \Psi-\frac{\tilde{R}}{8} \Psi+\frac{1}{32} \Psi^{5-2 a} \tilde{N}^{-2}(\tilde{L} \beta)_{i j}(\tilde{L} \beta)^{i j}-\frac{1}{12} K^{2} \Psi^{5}=0, \\
\tilde{\Delta} \beta^{i}+\frac{1}{3} \tilde{D}^{i} \tilde{D}_{k} \beta^{k}+\tilde{R}_{k}^{i} \beta^{k}-\tilde{N}^{-1}(\tilde{L} \beta)^{i k} \tilde{D}_{k} \tilde{N}-(a-6) \Psi^{-1}(\tilde{L} \beta)^{i k} \tilde{D}_{k} \Psi=\frac{4}{3} \Psi^{a} \tilde{N} \tilde{D}^{i} K, \\
\tilde{\Delta} \tilde{N}+2(a+1) \tilde{D}^{k} \ln \Psi \tilde{D}_{k} \ln \tilde{N}+\tilde{N}\left[\frac{a}{8} \tilde{R}+\frac{a-4}{12} \Psi^{4} K^{2}+a(a+1) \tilde{D}^{k} \ln \Psi \tilde{D}_{k} \ln \Psi\right]-\frac{a+8}{32} \Psi^{4-2 a} \tilde{N}^{-1}(\tilde{L} \beta)_{i j}(\tilde{L} \beta)^{i j} \\
=\Psi^{4-a} \beta^{k} \tilde{D}_{k} K .
\end{gathered}
$$

Different choices of the rescaling exponent $a$ have been considered in the literature: $a=6$ for defining the conformal lapse in Refs. [14,15], $a=-1$ is used in the numerical implementations of Refs. [4-6], and $a=-2$ in Ref. [9]. The problem we address in this work is the numerical study of the elliptic system (12), when completed with the inner boundary conditions in Sec. II.

\section{B. Inner boundary conditions}

Inserting the conformal decompositions ${ }^{4}(9)$ in the geometrical boundary conditions (3), one finds

$$
4 \tilde{s}^{i} \tilde{D}_{i} \Psi+\tilde{D}_{i} \tilde{s}^{i} \Psi=-\frac{\Psi^{3-a}}{2 \tilde{N}}(\tilde{L} \beta)_{i j} \tilde{s}^{i} \tilde{s}^{j}+\frac{2}{3} \Psi^{3} K,
$$

from the vanishing expansion [2,11,21-23], and

$$
\begin{aligned}
& {\left[{ }^{2} \tilde{D}^{i} V^{j}+{ }^{2} \tilde{D}^{j} V^{i}-\left({ }^{2} \tilde{D}_{k} V^{k}\right) \tilde{q}^{i j}\right]} \\
& +\left[\left(\tilde{N} \Psi^{a-2}-\tilde{b}\right)\left(\tilde{H}^{i j}-\frac{1}{2} \tilde{q}^{i j} \tilde{H}\right)\right]=0,
\end{aligned}
$$

from the vanishing of the shear [4,11], where $\beta^{i}=\tilde{b} \tilde{s}^{i}-$ $V^{i}, \tilde{H}_{i j}=q_{i}^{k} \tilde{D}_{k} \tilde{s}_{j}$ is the (conformal) extrinsic curvature of $\mathcal{S}$ as a hypersurface in $\Sigma$ (with trace $\tilde{H}=q^{k l} \tilde{H}_{k l}$ ), and we have used $\dot{\tilde{\gamma}}^{i j}=0$. If one enforces boundary condition (7), the second bracket is zero, and the vanishing of the first line of Eq. (14) characterizes $V^{i}$ as a conformal Killing vector of $\left(\mathcal{S}, \tilde{q}_{i j}\right)[4,11]$. This provides a Dirichlet condition for $V^{i}$ once the conformal isometry has been chosen.

In addition to the geometrical or numerical motivations for the boundary conditions, we must also consider at this point the analytical well-posedness of the elliptic problem. A procedure to establish the uniqueness of the solution of an elliptic equation (even though not straightforwardly generalizable to an elliptic system) consists in making use of a maximum principle [22,23]. This involves the control of the convexity of the functions we are solving for (in our case depending on the choice of the exponent $a$ ), and, in particular, on the signs of their radial derivatives at the boundaries. From condition (13), control of $\tilde{s}^{i} \tilde{D}_{i} \Psi$ on the horizon demands the control of the sign and size of $(\tilde{L} \beta)_{i j} \tilde{s}^{i} \tilde{S}^{j}$, something problematic if only the Dirichlet condition (7) is imposed. An alternative condition proposed in [12], in the context of a conformal transverse

\footnotetext{
${ }^{4}$ We also introduce the rescaled induced metric $\tilde{q}_{i j}$ on $\mathcal{S}: \tilde{q}_{i j}=$ $\Psi^{4} q_{i j}=\tilde{\gamma}_{i j}-\tilde{s}_{i} \tilde{s}_{j}$, with $\tilde{s}^{i}=\Psi^{2} s^{i}$.
}

traceless (CTT) decomposition, consists in prescribing ${ }^{5}$ $\Psi^{6} \cdot K_{i j} s^{i} s^{j}$ to satisfy

$$
-\tilde{H}<\Psi^{6} \cdot K_{i j} s^{i} s^{j} \leq 0 .
$$

Using the notation $f_{1} \equiv \Psi^{6} \cdot K_{i j} s^{i} s^{j}$, this is enforced as $2 \tilde{s}^{k} \tilde{D}_{k} \tilde{b}-\tilde{b} \tilde{H}=3 N \Psi^{-6} f_{1}-{ }^{2} \tilde{D}_{k} V^{k}-2 V^{k} \tilde{D}_{\tilde{s}} \tilde{s}_{k}-N K$.

If condition (7) is not imposed, the vanishing of the shear must be fulfilled by choosing, in addition to $V^{i}$ as a conformal Killing symmetry, free data such that the traceless part of $\tilde{H}_{i j}$ vanishes (umbilical condition). More generally one could solve condition (14) as an equation for $V^{i}$ [13]. Extending the well-posedness analysis from the CTT to the CTS case is an important issue. Difficulties are twofold. On the one hand, as pointed out in [24] (see also [25]), signs in Eqs. (12) for the $(\tilde{L} \beta)_{i j}(\tilde{L} \beta)^{i j}$ terms in the $\Psi$ and $\tilde{N}$ equations are problematic for applying a maximum principle argument. No obvious choice of $a$ cures the problem. On the other hand, the inclusion of new nonlinear coupled boundary conditions for $\tilde{N}$ makes the analytical problem even harder. However, a strong motivation for boundary condition (15) follows from its close relation with the characterization of $\mathcal{S}$ as a future trapped surface, i.e. with $\theta_{(k)} \leq 0$, via the identity

$$
K_{i j} s^{i} s^{j}-K=\frac{\theta_{(\ell)}}{2 N}+N \theta_{(k)}=N \theta_{(k)}=\theta_{(\hat{k})}
$$

where $\hat{k}^{\alpha}:=\frac{1}{2}\left(n^{\alpha}-s^{\alpha}\right)$. More importantly, condition (15) is not specifically tied to quasiequilibrium; in fact, $\theta_{(k)} \leq 0$ is part of the very definition of dynamical horizons, in order to guarantee the horizon area increase law [7]. Moreover, such a condition on the sign of $\theta_{(k)}$ permits the exclusion of certain pathologies in the evolution of the horizon, e.g. the appearance of self-intersections [26] of the surface $\mathcal{S}_{t}$, something to be avoided during the nonmerger phase of the black hole evolution. The present quasiequilibrium context offers a controlled test bed for studying this condition. Numerical techniques seem to be an appropriate tool for a first analysis of this problem.

\footnotetext{
${ }^{5}$ Notation here differs from that in Ref. [12]. Objects with tilde in Ref. [12] represent physical quantities, whereas here they refer to conformal counterparts according to (9).
} 


\section{NUMERICAL RESULTS}

We make use of two independent codes for solving Eqs. (12), both using pseudospectral methods: the first one employs the elliptic solvers described in Refs. [2729] and implemented in the C ++ library LORENE [30]. The second code has been specifically designed for the purpose of this paper. It uses a single domain technique for solving elliptic boundary value problems in the exterior of an excised spherical shell.

In order to determine the elliptic system, we complete Eqs. (12) with a specific combination of five of the boundary conditions reviewed in the previous section. Different possibilities arise, but all of them must incorporate Eqs. (13) and (14). For concreteness, in this paper we will restrict ourselves to axially symmetric excised spheres (with azimuthal symmetry $\phi^{i}$ ), and impose as a Dirichlet boundary condition for the tangential part of the shift: $V^{i}=\Omega_{\mathrm{o}} \cdot \phi^{i}$, with $\Omega_{\mathrm{o}}$ a constant [vanishing of the shear requires then appropriate additional boundary conditions or suitable free initial data in order to cancel the second line in Eq. (14)]. Equations (13) and (14) fix three of the five boundary conditions. The remaining two will be chosen among Eqs. (6)-(8) and (16). At this stage of the analysis it is methodologically useful to interpret each condition as associated with a specific equation of the system, even if this makes no strict sense due to the (nonlinear) coupled character of the boundary conditions. Table I summarizes this strategy, followed in Refs. [2,4,11,12]. The numerical implementations show that, keeping the conditions fixed for $\Psi$ and $V^{i}$, the different combinations of conditions for $\tilde{b}$ and $N$ actually lead to the existence of solutions, at least for appropriate ranges of the free parameters (and independently of $\tilde{\gamma}_{i j}$ and $K$ ). The solutions are generically unique or finite in number, and only very particular (nongeneric) choices of the functions $f_{1}$ and $f_{2}$ lead to the appearance of an infinite number of solutions.

\section{A. Combination $(b=N, \kappa=$ const $)$}

The main goal of this section is to assess the existence and uniqueness properties of those solutions to the CTS elliptic system which implement the constant surface gravity prescription (6), together with the adaptation (7) of the coordinate system to the horizon. Note that this set of boundary conditions realizes the vanishing of the shear for any choice of $\tilde{\gamma}_{i j}$. As a secondary objective, and fol-

TABLE I. Methodological assignment of boundary conditions to constrained fields. We keep fixed conditions for $\Psi$ and $V^{i}$ and study different combinations of the conditions for $\tilde{b}$ and $N$.

\begin{tabular}{cccc}
\hline \hline$\Psi$ & $V^{i}$ & $\tilde{b}=\beta^{k} \tilde{s}_{k}$ & $N$ \\
\hline$\theta_{(\ell)}=0$ & $V^{i}=\Omega_{\mathrm{o}} \cdot \phi^{i}$ & $b=N$ & $\kappa=$ const \\
& & $\Psi^{6} \cdot K_{i j} s^{i} s^{j}=f_{1}$ & ${ }^{2} D_{k} \Omega^{k}=f_{2}$ \\
\hline \hline
\end{tabular}

lowing the proposal in Ref. [11], we also study the wellposedness of employing the preferred notion of horizon time evolution that emerges from the isolated horizon Hamiltonian analysis, in order to fix the foliation by imposing the canonical choice $\kappa_{\mathrm{o}}=\kappa_{\mathrm{Kerr}}(a, J)$. In this case, condition (6) becomes

$$
s^{i} D_{i} N-N K_{i j} s^{i} s^{j}=\kappa_{\mathrm{Kerr}}(a, J),
$$

where $\kappa_{\text {Kerr }}(a, J)$ is a functional of $\left(\Psi, \beta^{i}, N\right)$. We start by considering the spherically symmetric case, $\Omega_{\mathrm{o}}=0$. First we note that the elliptic system implementing condition (18) admits an infinite number of solutions, since maximal slicings of Schwarzschild provide the 2-parameter Estabrook-Wahlquist family of solutions [31], as pointed out in Ref. [4]. Fixing the coordinate radius of the excised sphere only fixes one of these parameters, leading to a degenerate problem. The very nature of this degeneracy suggests the way out, since setting the surface gravity to a given constant $\kappa_{\mathrm{o}}$ fixes the representative of the EstabrookWahlquist family. As a code test, we have confirmed numerically that the system $\left(b=N, \kappa=\kappa_{\mathrm{o}}\right.$ ) determines a unique solution, leading to a well-posed problem. In addition, the a posteriori evaluation of the quantity $\kappa_{\text {Kerr }}(a, J)$ on the constructed solution, results in $\kappa_{\text {Kerr }}(a, J)=\kappa_{\mathrm{o}}$. This is in agreement with the degeneracy of the system $\left[b=N, \kappa_{\mathrm{o}}=\kappa_{\mathrm{Kerr}}(a, J)\right]$, meaning that the operators on the left- and right-hand sides of Eq. (18) become identical on the space of solutions of this elliptic system. We conclude that, in the spherically symmetric case, a well-posed problem is actually defined by imposing that the coordinate system be adapted to the horizon $(b=$ $N$ ) together with prescribing a given constant value for the surface gravity. In this system, the Hamiltonian canonical value for the surface gravity $\kappa=\kappa_{\text {Kerr }}(a, J)$ cannot be imposed as a boundary condition, but it is actually recovered in the solution. Regarding the range of possible values of $\kappa_{\mathrm{o}}$, this parameter is bounded by below, $\kappa_{\mathrm{o}} \geq 1 / 8$. In particular, this lower bound is associated with the vanishing of the lapse function on the horizon. No maximum value exists for $\kappa_{\mathrm{o}}$. However, the quadratic growth of the lapse when $\kappa_{\mathrm{o}}$ increases, makes $N$ reach very large values very rapidly. For this reason the convergence of our codes is limited to a maximum value of $\kappa_{\mathrm{o}}$.

More generally, we have also found that the system $(b=$ $N, \kappa=\kappa_{\mathrm{o}}$ ) is well-posed in the rotating case, $\Omega_{\mathrm{o}} \neq 0$. On the other hand, the situation regarding the canonical value of the surface gravity changes with respect to the spherically symmetric case: the a posteriori evaluation of $\kappa_{\text {Kerr }}(a, J)$ does not provide identically $\kappa_{\mathrm{o}}$. This means that, in the case where there exists a solution to the system $\left[b=N, \kappa=\kappa_{\text {Kerr }}(a, J)\right]$, the problem is not infinitely degenerate. In particular such a solution only exists if, when screening the solutions obtained by prescribing $\kappa$, there is a value $\kappa_{\mathrm{o}}$ for which $\kappa_{\text {Kerr }}(a, J)=\kappa_{\mathrm{o}}$ holds. Our numerical implementations seem to rule out this possibil- 


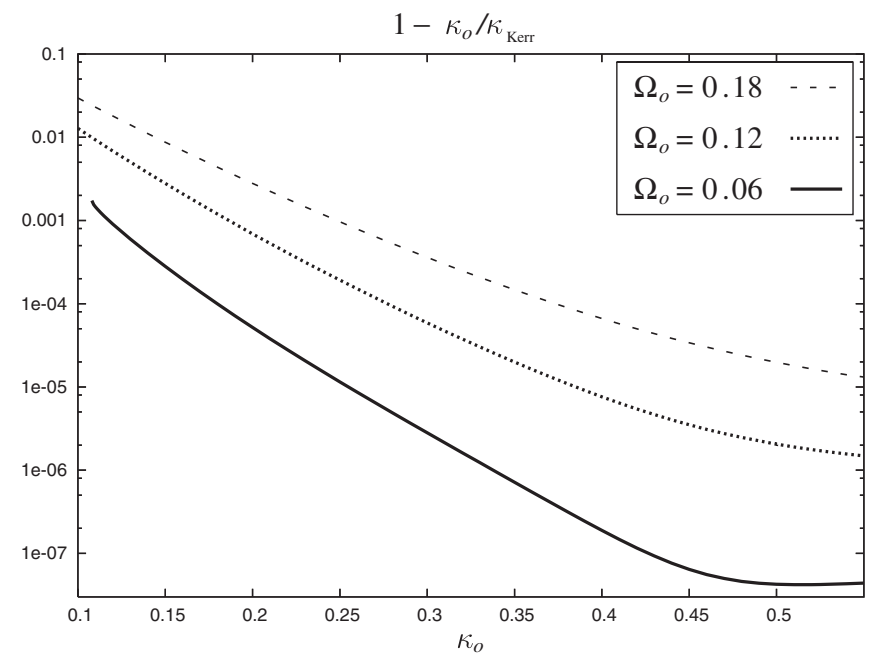

FIG. 1. Relative difference between $\kappa_{\text {Kerr }}(a, J)$ and $\kappa_{\mathrm{o}}$, as a function of $\kappa_{\mathrm{o}}$, for solutions to $\left(b=N, \kappa=\kappa_{\mathrm{o}}\right)$ for different values of $\Omega_{\mathrm{o}}$ in the rotating case.

ity, as illustrated by Fig. 1. This figure (implemented in conformal flatness, for concreteness) shows the relative difference between the prescribed value of $\kappa_{\mathrm{o}}$, and the evaluation of $\kappa_{\text {Kerr }}(a, J)$ in the constructed solution. As in the spherically symmetric case, a minimum value for $\kappa_{\mathrm{o}}$ is found, whereas the growth of the lapse limits the upper values we can numerically implement. In this range, and for $\Omega_{\mathrm{o}}=0.06$, a nonvanishing minimum difference between $\kappa_{\mathrm{O}}$ and $\kappa_{\text {Kerr }}(a, J)$ is actually found. A similar behavior is found for bigger values of $\Omega_{\mathrm{o}}$, even though the numerical limitations prevent us from determining the minimum.

Therefore, in the rotating case $\Omega_{\mathrm{o}} \neq 0$, we conclude that: (i) given a fixed constant $\kappa_{\mathrm{o}}$, the system $(b=N, \kappa=$ $\kappa_{\mathrm{o}}$ ) determines a unique solution of the CTS elliptic system; and (ii) the Hamiltonian canonical choice $[b=N$, $\left.\kappa=\kappa_{\text {Kerr }}(a, J)\right]$ seems to lead to an ill-posed problem, but because of the nonexistence of solutions rather than because of the presence of infinitely many, as in the spherically symmetric case.

\section{B. Extending the well-posedness analysis from CTT to CTS: $\Psi^{\mathbf{6}} \cdot K_{i j} s^{s^{i} s^{j}}$ versus $\theta_{(\hat{k})}$ prescription}

One of the main goals of this work is the study of condition (15) and (16) in the CTS system. In Ref. [12] it was shown that this condition, together with (13) and (14), defined a well-posed problem in the CTT construction of initial data. A natural question is to study how this result generalizes to the elliptic system enlarged with an additional equation for the lapse. In a strict sense, this question is not properly formulated, since its answer will depend on the fifth boundary condition "for the lapse." The aim here is rather to assess if a qualitative conclusion (independent of the details of the fifth boundary condition), can be formulated about the possible range of values of $\Psi^{6}$.
$K_{i j} s^{i} s^{j}$, with a focus on the negative ones. Our intention is not to perform a merely formal (numerical) extension of the CTT analytical result: we are ultimately motivated by probing some technical issues that will arise in the dynamical regime of the horizon. Indeed, given the relation with $\theta_{(\hat{k})}$ via Eq. (17), and the need to control the sign of $\theta_{(\hat{k})}$ in a dynamical horizon, it is fundamental to know the range of values we can actually prescribe on $\mathcal{S}_{t}$.

(a) Nonrotating case: $\Omega_{\mathrm{o}}=0$. Let us first consider the spherically symmetric case. In addition to the boundary conditions in the first two columns of Table I, and in order to probe the possible values of $\Psi^{6} \cdot K_{i j} s^{i} s^{j}$, let us prescribe $K_{i j} s^{i} s^{j}=\lambda<0$, with $\lambda$ a negative constant on $\mathcal{S}$. We then complete the elliptic system with different boundary conditions "for the lapse," and for each of them we screen the (negative) values of $\lambda$ for which we can construct a solution. Finally for each found solution, i.e. for each value of $\lambda$, we plot the dimensionless quantity $\left(\Psi^{6} \cdot K_{i j} s^{i} s^{j}\right) / \tilde{H}$ against $\left(\theta_{(\hat{k})}\right) / \tilde{H}$. In Fig. 2 we present the resulting curve where, for completeness, an extension to positive values of $\lambda$ has been included. The curve proves to be independent of the "lapse" boundary condition and, even though numerically we can only reach finite values of $\lambda$, in the spherically symmetric case an analytical expression can in fact be obtained (see the appendix). For this reason, a compactification with the function arctan has been implemented for plotting Fig. 2 .

The existence in Fig. 2 of a minimum $\delta_{\min }$ for $\Psi^{6}$. $K_{i j} s^{i} s^{j}$ and of an asymptotic value

$$
\delta_{\text {asym }}=\lim _{\lambda \rightarrow-\infty} \Psi^{6} \cdot K_{i j} s^{i} s^{j}=-2 \tilde{H}<0
$$

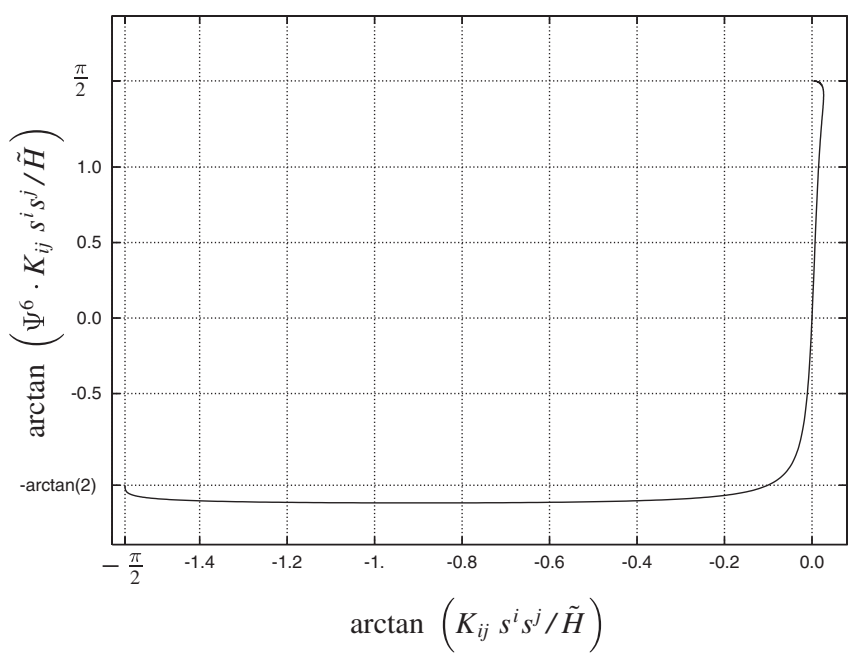

FIG. 2. Numerical analysis of condition (15). Values of $\theta_{(\hat{k})} / \tilde{H}=K_{i j} s^{i} s^{j} / \tilde{H}$ and $\Psi^{6} \cdot K_{i j} s^{j} s^{j} / \tilde{H}$ in the spherically symmetric case, after compactification by means of the arctan function. 
show that: (i) the possible negative values of $\Psi^{6}$. $K_{i j} s^{i} s^{j}$ are bounded by below: $\delta_{\min } \leq \Psi^{6} \cdot K_{i j} s^{i} s^{j}$; and (ii) there is a range $\delta_{\text {asym }} \leq \Psi^{6} \cdot K_{i j} s^{i} s^{j} \leq 0$ for which the prescription of the value of $\Psi^{6} \cdot K_{i j} s^{i} s^{j}$ determines a unique solution of the elliptic system, exactly as was concluded analytically in the CTT case. Let us also note that uniqueness is lost when the value $\Psi^{6} \cdot K_{i j} s^{i} s^{j}$ is prescribed between the values $\delta_{\text {min }}<\Psi^{6} \cdot K_{i j} s^{i} s^{j}<\delta_{\text {asym }}$, for which two solutions exist. This is just another example of the nonuniqueness issue associated with solutions of the CTS equations, already pointed out in Ref. [24]. This is in contrast to the prescription of a negative nonconformally rescaled $K_{i j} s^{i} s^{j}=\theta_{(\hat{k})}$ : in the spherically symmetric case, for each negative value of $\theta_{(\hat{k})}$ (not bounded by below) there exists a unique solution. This suggests $\theta_{(\hat{k})}$ as the function to be prescribed on $\mathcal{S}$, since no knowledge additional to the sign of $\theta_{(\hat{k})}$ is needed for consistency (see below).

The situation is reversed for positive values of $\lambda$, where only small values for $\theta_{(\hat{k})}$ can be prescribed, leading always to a degenerate solution. An appropriate parameter is then $\Psi^{6} \cdot K_{i j} s^{i} s^{j}$. However, these solutions do not describe a future marginally trapped surface and their interest in the present context is only formal.

(b) Rotating case: $\Omega_{\mathrm{o}} \neq 0$. The independence of the curve $\Psi^{6} \cdot K_{i j} s^{i} s^{j}$ vs $\theta_{(\hat{k})}$ on the "lapse boundary condition" disappears in the rotating case. This is illustrated in Figs. 3-5, where different boundary

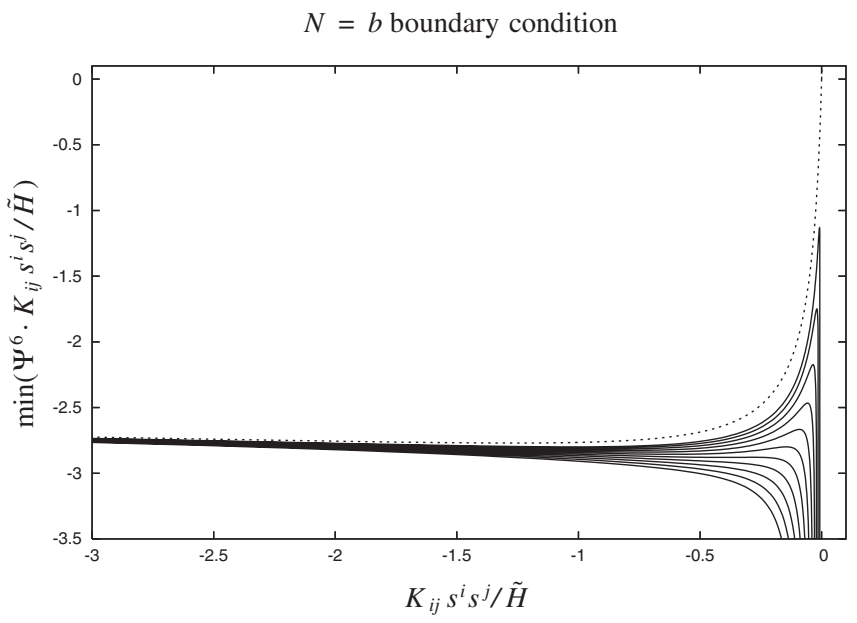

FIG. 3. Values of $\Psi^{6} \cdot K_{i j} s^{i} s^{j} / \tilde{H}$ and $\theta_{(\hat{k})} / \tilde{H}$, for boundary conditions $K_{i j} s^{i} s^{j}=\lambda, \quad b=N$ and $\Omega_{\mathrm{o}}=0,0.10,0.11,0.12$, $\ldots, 0.20$. Curves depart from the spherically symmetric case (dotted curve corresponds to $\Omega_{\mathrm{o}}=0$ ) as the rotating parameter increases. Solutions exist for every prescribed $\theta_{(\hat{k})}$ and are unique.
$K_{i j} s^{i} s^{j}=\lambda, N=0.2$

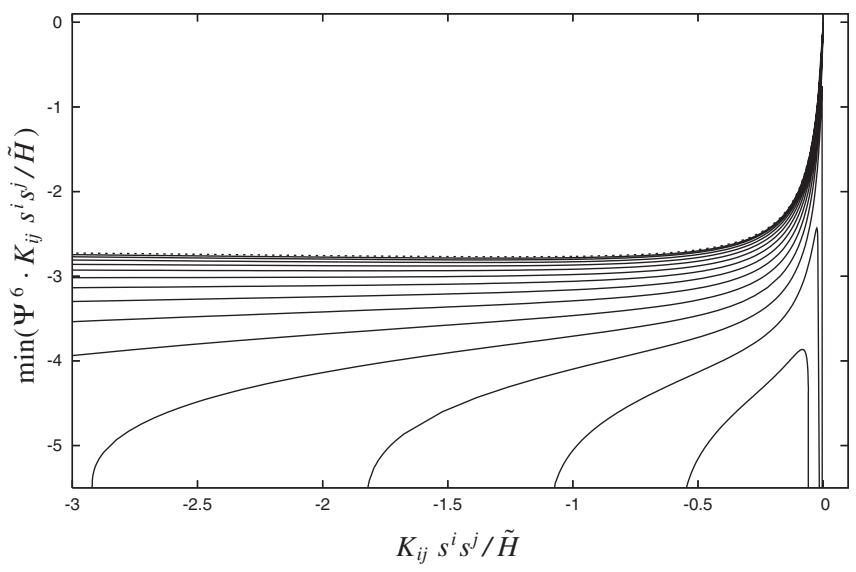

FIG. 4. Values of $\Psi^{6} \cdot K_{i j} s^{i} s^{j} / \tilde{H}$ and $\theta_{(\hat{k})} / \tilde{H}$, for boundary conditions $K_{i j} s^{i} s^{j}=\lambda, \quad N=0.2$ and $\Omega_{\mathrm{o}}=0.00,0.01$, $0.02, \ldots, 0.15$. If the rotating parameter is smaller than $\Omega_{\mathrm{o}} \approx$ 0.12620 , the curves reach the coordinate origin, otherwise they diverge to $-\infty$ as $\theta_{(\hat{k})} / \tilde{H} \rightarrow 0$.

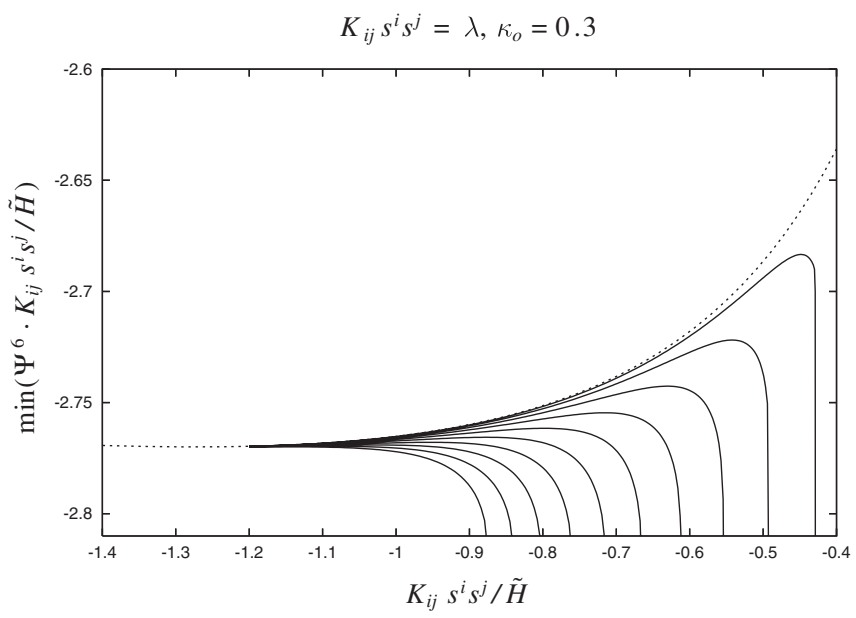

FIG. 5. Values of $\Psi^{6} \cdot K_{i j} s^{i} s^{j} / \tilde{H}$ and $\theta_{(\hat{k})} / \tilde{H}$, for boundary conditions $K_{i j} s^{i} s^{j}=\lambda, \kappa_{\mathrm{o}}=0.3$ and $\Omega_{\mathrm{o}}=0.00,0.02,0.04$, $\ldots, 0.18,0.20$. Curves with $\Omega_{\mathrm{o}} \neq 0$ do not pass through the origin: conclusions in Ref. [12] do not extend to the rotating case. On the other hand, $\Omega_{\mathrm{o}} \neq 0$ curves become indistinguishable of the spherically symmetric curve (dotted line) for sufficiently negative values of $K_{i j} s^{i} s^{j}$.

conditions for $N$ have been implemented ${ }^{6}$ (due to the angular dependence, we plot now the minimum of $\left.\Psi^{6} \cdot K_{i j} s^{i} s^{j}\right)$.

The most dramatic qualitative change in the rotating case is the existence of a certain value of $\Omega_{\mathrm{o}}$ such that, for bigger values of this rotation parameter, the curve does not pass through the origin (in the case of

\footnotetext{
${ }^{6}$ In order to implement quasiequilibrium, and according to Eq. (14), we restrain here to a flat $\tilde{\gamma}_{i j}$ (this guarantees the umbilical condition $\tilde{H}^{i j}-\frac{1}{2} \tilde{q}^{i j} \tilde{H}=0$ ).
} 
Figs. 3 and 5 this is true for any $\Omega_{0} \neq 0$ ). Arbitrarily negative values of $\Psi^{6} \cdot K_{i j} s^{i} s^{j}$ are found for small values of $\theta_{(\hat{k})}$. Therefore in this range of $\Omega_{\mathrm{o}}$, and in contrast to the spherically symmetric case, the negative values of $\Psi^{6} \cdot K_{i j} s^{i} s^{j}$ are only bounded from above. This shows that conclusions in Ref. [12] for the CTT case do not extend to the CTS one for arbitrary values of $V^{i}=\Omega_{0} \cdot \phi^{i}$. Given the variety of behaviors in Figs. 3-5, it is difficult to extract generic conclusions, i.e. independent of the boundary conditions on $N$, about the possible values of $\theta_{(\hat{k})}$ and $\Psi^{6} \cdot K_{i j} s^{i} s^{j}$. Our numerical simulations do not provide a complete understanding, but rather some restricted insight, of the boundary condition properties in the case of a rotating black hole.

Having stated this clearly, we highlight Fig. 3 corresponding to the interpretation of boundary condition (7) as the lapse boundary condition, $N=b$. In this case, we can actually formulate a concrete statement about the parameter to be prescribed: for all values of $\Omega_{\mathrm{o}}$, there exists a (small) negative value $K_{i j} s^{i} s^{j}=\Delta_{\Omega_{\mathrm{o}}} \leq 0$ such that the prescription of $\theta_{(\hat{k})}$ to $\lambda<\Delta_{\Omega_{\mathrm{o}}}$ determines a unique solution. It is very difficult to determine numerically if $\Delta_{\Omega_{\mathrm{o}}} / \tilde{H}$ is actually zero or a very small value depending on $\Omega_{\mathrm{o}}$. At this value $K_{i j} s^{i} s^{j}=\Delta_{\Omega_{\mathrm{o}}}$ the conformal factor seems to have a pole and the quantity $\Psi^{6} \cdot K_{i j} s^{i} s^{j}$ diverges to negative values. As a consequence, curves in Fig. 3 for $\Omega_{\mathrm{o}} \neq 0$ do not reach the origin, as pointed out above, changing the qualitative behavior discussed in the spherically symmetric case. Still there exists a critical value $\Omega_{\text {crit }}(\approx 0.15)$ such that, in the range $\Omega_{\mathrm{o}}<\Omega_{\text {crit }}$, all curves present a local minimum $\delta_{\min }$ and an asymptotic value $\delta_{\text {asym }}$ at $K_{i j} s^{i} s^{j} \rightarrow-\infty$. However, uniqueness (if existence at all) is lost when prescribing $\Psi^{6} \cdot K_{i j} s^{i} s^{j}>$ $\delta_{\text {asym }}$; because of the negative divergence of $\Psi^{6}$. $K_{i j} s^{i} s^{j}$, either there are two or no solutions. The ultimate reason for focusing on this "fifth" boundary condition, is that it presents some geometrical/ physical advantages with respect to the other ones: (i) it implements the NEH condition for any choice of $\tilde{\gamma}_{i j}$ without the need of relying on the umbilical condition (see footnote 6); and (ii) by enforcing the evolution vector $t^{\alpha}$ to be tangent to $\mathcal{H}$, which is in quasiequilibrium, a coordinate system is chosen on the horizon in which no time-dependence is artificially introduced as a gauge effect (see also footnote 3 for actual physical consequences in the binary case).

We conclude that $\Psi^{6} \cdot K_{i j} s^{i} s^{j}$ is not an appropriate function to be prescribed in a CTS approach. The present numerical analysis suggests that adaptation of the coordinate system to the horizon (boundary condition $b=N)$ and the prescription of $\theta_{(\hat{k})}$ to a sufficiently negative value determines a unique solution. This is relevant information for the dynami$\mathrm{cal}^{7}$ case, in particular in a constrained evolution scheme in which the elliptic system (12) is solved at each time step (see Ref. [9]).

\section{Prescription of the divergence of $\boldsymbol{\Omega}_{\boldsymbol{i}}$}

Since the solution $\ln N_{0}$ of Eq. (8) is defined up to a constant on $\mathcal{S}$, the value $N=C \cdot e^{\ln N_{0}}$ provides a Dirichlet condition for the lapse, which takes into account the manner in which $\mathcal{S}$ is embedded in the spacetime and permits one to control the magnitude of $N$ via the free choice of the constant $C$. The multiplicative character of the latter is a good feature on numerical grounds. Of course, condition (8) must be completed with a choice of ${ }^{2} D^{k} \Omega_{k}$ (an arbitrary function on $\mathcal{S} \approx S^{2}$ with vanishing $\ell=0$ mode). This makes this condition an effective one, in the sense discussed in Ref. [4] for "lapse boundary conditions." However, for specific problems natural choices exist, e.g. ${ }^{2} D^{k} \Omega_{k}=0$ for perturbations of the spherically symmetric case (see [7,13] for a Pawlowski gauge, motivated by KerrSchild-like slicings). More importantly, a part of the extrinsic geometry is incorporated into this boundary condition, which thus goes beyond a merely numerically convenient choice. Once ${ }^{2} D^{k} \Omega_{k}(=0)$ has been chosen, the slicing is completely fixed, and all the freedom is reduced to a single constant $C$ that controls the rate of motion across the slicing. We have numerically verified the existence of unique solutions when combining this condition with the ones in the third column of Table I.

\section{General comments}

Other possible combinations. Table I presents an association between boundary conditions and constrained fields. Even though this can be useful to organize the discussion, it must again be emphasized that this is only a methodological option. Insisting on such an association can be misleading and can obscure some useful choices. As an example, since condition (13) provides an expression for $K_{i j} s^{i} s^{j}$, it could be interpreted as a condition ${ }^{8}$ for $\tilde{b}$ via Eq. (16). Then, interpreting $b-N=0$ as a condition for $N$, we can think of prescribing a value for $\Psi$ on $\mathcal{S}$ by a Dirichlet condition. This particular example has two relevant applications: (i) it permits one to prescribe the area of $\mathcal{S}$ (since the conformal part is a free data) and (ii) it provides an alternative strategy for the analytical study of the well-posedness of the marginally trapped condition (Refs. [22,23] focus on the control of the radial derivative

\footnotetext{
${ }^{7}$ In the dynamical case, the boundary condition $b=N$ is generalized by solving an elliptic equation on $\mathcal{S}$; see $[32,33]$.

${ }^{8}$ In particular, together with (14) as a condition on $V^{i}$, this means that nonexpanding conditions can be fully fulfilled by an appropriate choice of the shift.
} 
of $\Psi$ in order to apply a maximum principle to the Hamiltonian constraint equation; the alternative of a Dirichlet condition for $\Psi$ shifts the focus to the momentum constraint).

Effective boundary conditions. At the end of the day, quasiequilibrium conditions (3) together with $b-N=0$ leave a single function to be specified on $\mathcal{S}$. Following $[2,4,11]$ this fifth function can be seen as a condition "for the lapse," either geometrically motivated or purely effective. But it must be underlined (see also in this sense the discussion in Sec. III.C of Ref. [4]), that it can be more generally interpreted as an effective condition on any scalar combination of fields, e.g. the conformal factor or ${ }^{2} D^{k} \Omega_{k}$.

Generic well-posedness. Finally, we have commented that combining conditions in Table I leads to well-posed problems for generic choices of $f_{1}$ and $f_{2}$. By this we mean that only for some critical $f_{1}$ and $f_{2}$ the problem admits an infinite number of solutions. For instance, condition $\mathcal{L}_{\ell} \theta_{(k)}=0$ in [2] can be recast as a prescription $f_{1}$ for $\Psi^{6}$. $K_{i j} s^{i} s^{j}$, and also as ${ }^{2} D^{k} \Omega_{k}=f_{2}$. If these particular expressions are used for conditions (16) or (8) in the spherically symmetric case, this leads to an ill-posed problem, as shown in Ref. [4]. By generic we mean here that a small perturbation of these critical cases makes the problem well-posed.

\section{CONCLUSIONS}

This work represents the numerical counterpart of Refs. [11-13], where isolated horizon boundary conditions were proposed. As a first result, the prescription of a constant value $\kappa=\kappa_{\mathrm{o}}$ on an instantaneously nonexpanding horizon, using a coordinate system adapted to the horizon [i.e. conditions (3), (6), and (7)], defines a wellposed problem. If $\kappa$ is set to $\kappa_{\text {Kerr }}(a, J)$, as proposed in [11], the problem is degenerate (infinite number of solutions) in the spherically symmetric case and admits no solution when rotation is introduced. The only freedom in this system is the choice of the constant $\kappa_{\mathrm{o}}$. The $\kappa=$ constant condition does not enforce a quasiequilibrium restriction on the geometry of the horizon. This means that it does not increase the physical degree of stationarity of the associated initial data (consistently with [4], where it is shown that physical quantities do not depend on the chosen lapse boundary condition). Its interest is rather in the evolution of such initial data, since it provides a slicing where the lapse function must be initially time independent, something desirable numerically.

Second, the results in Ref. [12] on the prescription of $\Psi^{6} \cdot K_{i j} s^{i} s^{j}$ in the CTT system, do not extend straightforwardly to the CTS case, except in spherical symmetry. In this particular case, there exists a negative bound $\delta_{\text {asym }}$ such that for $\delta_{\text {asym }} \leq \Psi^{6} \cdot K_{i j} s^{i} s^{j} / \tilde{H} \leq 0$ there is a unique solution. There exists a second bound $\delta_{\min }$ such that the prescription $\delta_{\min }<\Psi^{6} \cdot K_{i j} s^{i} s^{j} / \tilde{H}<\delta_{\text {asym }}$ admits two degenerate solutions. For $\Psi^{6} \cdot K_{i j} s^{i} s^{j} / \tilde{H}<\delta_{\min }$ no solu- tion exists. In the rotating case, the strong dependence on the fifth boundary condition prevents us from deriving general bounds for $\Psi^{6} \cdot K_{i j} s^{i} s^{j}$. However, for the particular choice of a coordinate system adapted to the horizon ( $b=N$ condition), the prescription of $\theta_{(\hat{k})}$ to a sufficiently negative value guarantees the existence and uniqueness of a solution to the CTS elliptic system. Therefore, rather than $\Psi^{6} \cdot K_{i j} s^{i} s^{j}$, the parameter to be prescribed is $\theta_{(\hat{k})}$. This represents important information for the implementation of evolving black holes as regular future trapping/dynamical horizons $[7,8,26]$ in a constrained evolution scheme.

Finally we have underlined the fact that prescribing nonexpanding and adapted-coordinate-system conditions leave one free function to be specified on the horizon. Because of the (nonlinear) coupled nature of the boundary conditions, this fifth condition is not specifically related to a particular field and, even though it can be useful to interpret it as a lapse boundary condition, other choices (e.g. a Dirichlet condition on $\Psi$ ) can prove to be useful.

\section{ACKNOWLEDGMENTS}

The authors are grateful to S. Bonazzola, S. Dain, E. Gourgoulhon, B. Krishnan, G. Mena-Marugán, J. Novak, and D. Petroff for numerous discussions. J. L. J. acknowledges the support of the Marie Curie IntraEuropean Contract No. MEIF-CT-2003-500885 within the 6th European Community Framework Programme, and the hospitality of the Albert Einstein Institute.

\section{APPENDIX A: COMMENTS ON FIGURE 2}

In this appendix we derive some analytical relations that are valid for CTS data in the conformally flat, maximal, spherically symmetric case. In particular we prove that the graph displayed in Fig. 2 is independent of a specific choice of inner boundary conditions on $N$ and $\tilde{b}$ (the prescription $K_{i j} s^{j} s^{j}=\lambda$ determines one point in the curve), and give a parametric analytical expression through which this curve is defined.

(a) Independence of Fig. 2. In spherical symmetry, with $(r, \theta, \phi)$ being spherical coordinates in which the apparent horizon is located at $r=r_{H}$, the maximal slicing CTS Eqs. (12) [with $a=0$ ] reduce to:

$$
\begin{aligned}
\frac{d^{2} \Psi}{d r^{2}}+\frac{2}{r} \frac{d \Psi}{d r}+\frac{\Psi^{5}}{12 N^{2}} r^{2}\left[\frac{d}{d r}\left(r^{-1} \beta^{r}\right)\right]^{2} & =0, \\
\frac{d^{2}}{d r^{2}}\left(r^{-1} \beta^{r}\right)+\frac{d}{d r}\left(r^{-1} \beta^{r}\right)\left[\frac{4}{r}-\frac{d}{d r}\left[\log \left(N \Psi^{-6}\right)\right]\right] & =0, \\
\frac{d^{2}}{d r^{2}}(N \Psi)+\frac{2}{r} \frac{d(N \Psi)}{d r}-\frac{7 \Psi^{5}}{12 N} r^{2}\left[\frac{d}{d r}\left(r^{-1} \beta^{r}\right)\right]^{2} & =0,
\end{aligned}
$$

and the apparent horizon boundary condition (note that $\beta^{\theta}=\beta^{\phi}=0$ ) is given by 


$$
\left[\frac{d \Psi}{d r}+\frac{\Psi}{2 r}+\frac{r \Psi^{3}}{6 N} \frac{d}{d r}\left(r^{-1} \beta^{r}\right)\right]_{r=r_{H}}=0 .
$$

With the introduction of the compactified radial coordinate $s=r_{H} / r$, we get

$$
\begin{gathered}
s^{2} \Psi^{\prime \prime}+B^{\prime 2} \frac{\Psi^{5}}{12 N^{2}}=0, \\
B^{\prime \prime}-B^{\prime}\left(\frac{2}{s}+\log \left[N \Psi^{-6}\right]^{\prime}\right)=0, \\
s^{2}(N \Psi)^{\prime \prime}-7 B^{\prime 2} \frac{\Psi^{5}}{12 N^{2}}=0,
\end{gathered}
$$

and

$$
\left[\Psi^{\prime}-\frac{\Psi}{2}+\frac{\Psi^{3}}{6 N} B^{\prime}\right]_{s=1}=0,
$$

where $^{\prime}=d / d s$ and $\beta^{r}=B / s$. Equation (A4) can be solved explicitly:

$$
B^{\prime}=c \cdot s^{2} N \Psi^{-6},
$$

where $c$ is a constant of integration, closely related to $\Psi^{6} \cdot K_{i j} s^{i} s^{j}$ [see Eq. (16) and below]. Using (A7) we obtain

$$
\Psi^{\prime \prime}+\frac{c^{2}}{12} s^{2} \Psi^{-7}=0
$$

together with the boundary condition

$$
\left[\Psi^{\prime}-\frac{\Psi}{2}+\frac{c}{6 \Psi^{3}}\right]_{s=1}=0 .
$$

The system (A8) and (A9) uniquely defines a sequence of solutions $\Psi(s ; c)$ which is independent of a specific choice of inner boundary conditions for $B$ and $N$. Moreover, the horizon quantity (cf. Eq. (16) in the spherically symmetric case)

$$
\begin{aligned}
{\left[K_{i j} s^{i} s^{j}\right]_{r=r_{H}} } & =\left[\frac{2}{3 N}\left(\frac{d}{d r} \beta^{r}-\frac{1}{r} \beta^{r}\right)\right]_{r=r_{H}} \\
& =-\frac{2}{3 r_{H}} c \Psi_{H}^{-6},
\end{aligned}
$$

where

$$
\Psi_{H}=\Psi(s=1 ; c),
$$

is a function of $c$ alone. Thus also the graph displayed in Fig. 2 is independent of a specific choice of inner boundary conditions on $\beta^{r}$ and $N$. The prescription $K_{i j} s^{i} s^{j}=\lambda$ determines a point in this curve.

(b) Analytic representation of Fig. 2. In order to obtain explicit mathematical expressions that describe the graph displayed in Fig. 2, we consider the spatial metric as well as the extrinsic curvature of the family of time-independent maximal slicings of the Schwarzschild solution [31], i.e.

$$
\begin{aligned}
d s^{2}= & \left(1-\frac{R_{H}}{R}+C^{2} \frac{R_{H}^{4}}{R^{4}}\right)^{-1} d R^{2} \\
& +R^{2}\left(d \theta^{2}+\sin ^{2} \theta d \phi^{2}\right)
\end{aligned}
$$

and

$$
K_{j}^{i}=C \frac{R_{H}^{2}}{R^{3}}\left(\begin{array}{ccc}
-2 & 0 & 0 \\
0 & 1 & 0 \\
0 & 0 & 1
\end{array}\right),
$$

which gives in particular

$$
\left[K_{i j} s^{i} s^{j}\right]_{R=R_{H}}=-\frac{2 C}{R_{H}} .
$$

In these coordinates $(R, \theta, \phi)$, the radius of the black hole horizon is given by $R=R_{H}=2 M$, where $M$ is the black hole mass. The constant $C$ parametrizes the family of maximal slicings.

The coordinate transformation $r=r(R)$, leading to a conformally flat line element

$$
d s^{2}=\Psi^{4}\left[d r^{2}+r^{2}\left(d \theta^{2}+\sin ^{2} \theta d \phi^{2}\right)\right],
$$

is described by

$$
s=\exp \left(\int_{1}^{s} \frac{d \sigma}{\sigma \sqrt{1-\sigma+C^{2} \sigma^{4}}}\right),
$$

where

$$
s=\frac{r_{H}}{r} \quad \text { and } \quad S=\frac{R_{H}}{R} .
$$

The conformal factor $\Psi$ which satisfies the boundary value problem (A8) and (A9), can be obtained from the comparison of the line elements (A12) and (A15):

$$
\Psi^{2}=\frac{R}{r}=\frac{R_{H}}{r_{H}} \frac{s}{S} .
$$

As a consequence

$$
\Psi_{H}^{2}=\frac{R_{H}}{r_{H}},
$$

and by imposing the asymptotic boundary condition, $\lim _{S \rightarrow 0} \Psi=1$, we can write $\Psi_{H}^{2}$ in terms of the parameter $C$ :

$$
\begin{aligned}
\Psi_{H}^{2}(C & \geq 0) \\
& =\exp \left(\int_{0}^{1} \frac{d \sigma}{\sigma}\left[\frac{1}{\sqrt{1-\sigma+C^{2} \sigma^{4}}}-1\right]\right) .
\end{aligned}
$$

The formula (A20) only describes $\Psi_{H}$ for nonnegative values of $C$. An extension of this expression into the realm of negative $C$-values yields, for 


$$
\begin{aligned}
0>C>-3 \sqrt{3} / 16 & \\
\Psi_{H}^{2}(C<0)= & \exp \left(\int_{0}^{1} \frac{d \sigma}{\sigma}\left[\frac{1}{\sqrt{1-\sigma+C^{2} \sigma^{4}}}-1\right]\right. \\
& \left.+2 \int_{1}^{s_{1}(C)} \frac{d \sigma}{\sigma \sqrt{1-\sigma+C^{2} \sigma^{4}}}\right),(\mathrm{A} 21)
\end{aligned}
$$

where $s_{1}(C)$ is the real zero of

$$
f(s)=1-s+C^{2} s^{4},
$$

with

$$
1 \leq s_{1}(C) \leq \frac{4}{3} .
$$

Note that $\Psi_{H}^{2}(C)$ tends to $+\infty$ as $C \rightarrow-3 \sqrt{3} / 16$ since $f(s)$ has a double zero, $s_{1}=4 / 3$, in this limit. With (A14) and $\tilde{H}=2 / r_{H}$, we finally obtain the desired parametric description of the curve displayed in Fig. 2 [a parametrization in terms of $c$ in Eq. (A11) can be also obtained from the relation between $C$ and $c$ provided by Eqs. (A10) and (A14)]:

$$
\begin{aligned}
& \text { Abscissa: } K_{i j} s^{i} s^{j} / \tilde{H}=-C \Psi_{H}^{-2}, \\
& \text { Ordinate: } \Psi_{H}^{6} \cdot K_{i j} s^{i} s^{j} / \tilde{H}=-C \Psi_{H}^{4} .
\end{aligned}
$$

A particular consequence of this analysis is

$$
\lim _{C \rightarrow \infty} \Psi_{H}^{6} \cdot K_{i j} s^{i} s^{j}=-2 \tilde{H},
$$

providing the asymptotic value when $K_{i j} s^{i} s^{j} / \tilde{H} \rightarrow$ $-\infty$.
[1] E. Gourgoulhon, P. Grandclément, and S. Bonazzola, Phys. Rev. D 65, 044020 (2002).

[2] G. B. Cook, Phys. Rev. D 65, 084003 (2002).

[3] H. P. Pfeiffer, Ph.D. thesis, Cornell University, 2003.

[4] G. B. Cook and H. P. Pfeiffer, Phys. Rev. D 70, 104016 (2004).

[5] M. Ansorg, Phys. Rev. D 72, 024018 (2005).

[6] M. Caudill, G. B. Cook, J. D. Grigsby, and H. P. Pfeiffer, Phys. Rev. D 74, 064011 (2006).

[7] A. Ashtekar and B. Krishnan, Living Rev. Relativity 7, 10 (2004), http://www.livingreviews.org/lrr-2004-10.

[8] I. Booth, Can. J. Phys. 83, 1073 (2005).

[9] S. Bonazzola, E. Gourgoulhon, P. Grandclément, and J. Novak, Phys. Rev. D 70, 104007 (2004).

[10] G. Schäfer and A. Gopakumar, Phys. Rev. D 69, 021501(R) (2004).

[11] J. L. Jaramillo, E. Gourgoulhon, and G. A. Mena Marugán, Phys. Rev. D 70, 124036 (2004).

[12] S. Dain, J. L. Jaramillo, and B. Krishnan, Phys. Rev. D 71, 064003 (2005).

[13] E. Gourgoulhon and J.L. Jaramillo, Phys. Rep. 423, 159 (2006).

[14] J. W. York, Phys. Rev. Lett. 82, 1350 (1999).

[15] H. P. Pfeiffer and J.W. York, Phys. Rev. D 67, 044022 (2003).

[16] O. Dreyer, B. Krishnan, D. Shoemaker, and E. Schnetter, Phys. Rev. D 67, 024018 (2003).

[17] A. Ashtekar, C. Beetle, and J. Lewandowski, Phys. Rev. D 64, 044016 (2001).

[18] E. Gourgoulhon, Phys. Rev. D 72, 104007 (2005).
[19] A. Ashtekar, S. Fairhurst, and B. Krishnan, Phys. Rev. D 62, 104025 (2000).

[20] A. Ashtekar, C. Beetle, and J. Lewandowski, Classical Quantum Gravity 19, 1195 (2002).

[21] J. Thornburg, Classical Quantum Gravity 4, 1119 (1987).

[22] S. Dain, Classical Quantum Gravity 21, 555 (2004).

[23] D. Maxwell, Commun. Math. Phys. 253, 561 (2004).

[24] H. P. Pfeiffer and J. W. York, Phys. Rev. Lett. 95, 091101 (2005); H. Pfeiffer, in Proceedings of the Einstein Constraint Equations workshop, held at Isaac Newton Institute for Mathematical Sciences, Cambridge, England, 2005 (unpublished).

[25] T. W. Baumgarte, N. Ó Murchadha, and H. P. Pfeiffer, grqc/0610120; D. M. Walsh, gr-qc/0610129.

[26] W. Simon, in Proceedings of the XXIX Spanish Relativity Meeting, ERE, Palma de Mallorca, 2006 (in press).

[27] S. Bonazzola, E. Gourgoulhon, and J.-A. Marck, J. Comput. Appl. Math. 109, 433 (1999).

[28] P. Grandclement, S. Bonazzola, E. Gourgoulhon, and J.-A. Marck, J. Comput. Phys. 170, 231 (2001)

[29] P. Grandclement, E. Gourgoulhon, and S. Bonazzola, Phys. Rev. D 65, 044021 (2002).

[30] http://www.lorene.obspm.fr.

[31] F. Estabrook, H. Wahlquist, S. Christensen, B. DeWitt, L. Smarr, and E. Tsiang, Phys. Rev. D 7, 2814 (1973).

[32] D. M. Eardley, Phys. Rev. D 57, 2299 (1998).

[33] E. Gourgoulhon and J. L. Jaramillo (unpublished); Talk given at the New Frontiers in Numerical Relativity meeting, AEI-Golm, 2006 (unpublished). 